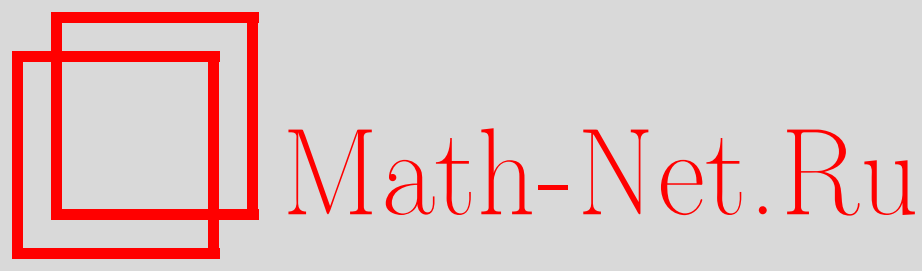

С. Д. Глызин, А. Ю. Колесов, Н. Х. Розов, К вопросу о реализуемости сценария развития турбулентности по Ландау, ТМФ, 2009, том 158, номер 2, 292-311

DOI: https://doi.org/10.4213/tmf6315

Использование Общероссийского математического портала Math-Net.Ru подразумевает, что вы прочитали и согласны с пользовательским соглашением http://www.mathnet.ru/rus/agreement

Параметры загрузки:

IP : 54.237 .59 .107

26 апреля 2023 г., 13:26:26

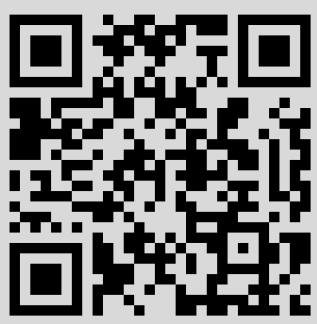




\section{К ВОПРОСУ О РЕАЛИЗУЕМОСТИ СЦЕНАРИЯ РАЗВИТИЯ ТУРБУЛЕНТНОСТИ ПО ЛАНДАУ}

Предложена феноменологическая модель развития турбулентности, представляющая собой нелинейное уравнение Клейна-Гордона, возмущенное неконсервативными добавками. С помощью сочетания аналитических и численных методов установлено, что переход к турбулентности в этом уравнении может происходить как по сценарию Ландау, так и в соответствии со сценарием Ландау-Селла. Первый из упомянутых сценариев связан, как известно, с каскадом бифуркаций устойчивых инвариантных торов все более высоких размерностей. Во втором случае мы имеем дело с хаотическим аттрактором, ляпуновская размерность которого при изменении некоторого управляющего параметра неограниченно растет.

Ключевые слова: сценарий Ландау, инвариантный тор, бифуркация, турбулентный аттрактор, хаос.

\section{1. ВВЕДЕНИЕ}

Динамическая теория развития турбулентности берет свое начало с классических работ Ландау [1] и Хопфа [2]. В первой из этих работ была выдвинута гипотеза о том, что возникновение турбулентности связано с последовательным усложнением динамики за счет появления устойчивых инвариантных торов все более высоких размерностей с квазипериодической обмоткой. Во второй работе построен достаточно простой пример динамической системы, в которой наблюдается описанный в работе [1] каскад бифуркаций инвариантных торов.

Гипотеза Ландау сыграла большую роль в процессе осознания природы турбулентности. В частности, развитые Ландау представления легли в основу известных гипотез Колмогорова о росте размерности аттракторов уравнений Навье-Стокса при увеличении числа Рейнольдса. Однако современные представления о турбулентности сложились во многом благодаря открытию феномена динамического хаоса [3]. K настоящему времени выработано несколько различных эвристических сценариев

* Ярославский государственный университет, Ярославль, Россия.

E-mail: glyzin@uniyar.ac.ru, kolesov@uniyar.ac.ru

${ }^{\dagger}$ Московский государственный университет им. М. В. Ломоносова, Москва, Россия. E-mail: rozov@rozov.mccme.ru 
перехода к турбулентности, среди которых следует отметить разрушение квазипериодических движений [4], [5], перемежаемость [6] и бесконечную последовательность бифуркаций удвоения периода [7]. Что же касается сценария Ландау, то его нельзя отнести к числу основных, поскольку аттрактор в виде незамкнутой плотной намотки на многомерном торе структурно неустойчив, т.е. он разрушается при малом изменении параметров системы. Тем не менее усложнение динамики по Ландау все же реализуется в некоторых специальных случаях. Для примера сошлемся на статью [8], где изучалась цепочка однонаправленно связанных ротаторов.

Итак, достаточно ясно, что рассматривать вопрос о реализуемости механизма развития турбулентности по Ландау в его первоначальном варианте не имеет смысла. В связи с этим всюду ниже, следуя идеям Селла [9], под сценарием Ландау мы будем понимать каскад бифуркаций

$$
T_{1} \rightarrow T_{2} \rightarrow \cdots \rightarrow T_{N} \rightarrow T_{N+1} \rightarrow \cdots
$$

устойчивых инвариантных торов $T_{N}, N \geqslant 1$, не требуя при этом квазипериодичности движений на каждом из них. Очевидно, что в таком расширенном варианте данный сценарий уже не является экзотическим. Более того, его можно связать с концепцией динамического хаоса, если в цепочке (1) каждый инвариантный тор $T_{N}$, начиная с некоторого номера $N_{0}$, заменить хаотическим аттрактором $A_{N}$ и предположить, что $d_{\mathrm{L}}\left(A_{N}\right) \rightarrow \infty$ при $N \rightarrow \infty$, где $d_{\mathrm{L}}-$ ляпуновская размерность. Так как при этом не исключен случай $A_{N} \subset T_{N}, N \geqslant N_{0}$, получившуюся в результате последовательность бифуркаций уместно назвать сценарием Ландау-Селла.

Дополнительным аргументом в пользу введенного термина служит тот факт, что принципиальная возможность сохранения хаотического аттрактора при бифуркации $T_{N} \rightarrow T_{N+1}$ была установлена в уже упоминавшейся статье Селла [9].

Цель настоящей работы - проиллюстрировать реализуемость сценариев Ландау и Ландау-Селла в рамках некоторой феноменологической модели. В качестве таковой рассматривается нелинейная краевая задача

$$
\begin{gathered}
u_{t t}-a^{2} u_{x x}+u+b u^{5}=\frac{\partial}{\partial t}\left(\varepsilon u+\nu u_{x x}\right)-u_{t} \int_{0}^{1}\left(u_{t}\right)^{2} d x, \\
\left.u\right|_{x=0}=\left.u\right|_{x=1}=0,
\end{gathered}
$$

где $a, b, \varepsilon, \nu$ - произвольные положительные параметры, $u=u(t, x)$ - скалярная вещественная функция.

Причины, по которым мы остановили свой выбор на модели (2), состоят в следующем. Во-первых, при ее построении за основу взято нелинейное уравнение Клейна-Гордона на отрезке $0 \leqslant x \leqslant 1$ с нулевыми граничными условиями Дирихле. Соответствующую краевую задачу

$$
u_{t t}-a^{2} u_{x x}+u+b u^{5}=0,\left.\quad u\right|_{x=0}=\left.u\right|_{x=1}=0
$$

можно рассматривать как некий аналог уравнений Эйлера идеальной несжимаемой жидкости, так как, в частности, она допускает закон сохранения

$$
\int_{0}^{1}\left(\left(u_{t}\right)^{2}+a^{2}\left(u_{x}\right)^{2}+u^{2}+b \frac{u^{6}}{3}\right) d x \equiv \text { const . }
$$


Во-вторых, при переходе от (3) к (2) мы добавили в правую часть уравнения КлейнаГордона слагаемые $\varepsilon u_{t}-u_{t} \int_{0}^{1}\left(u_{t}\right)^{2} d x$ и $\nu u_{t x x}$, которые в гидродинамической интерпретации соответствуют возбуждающей силе и вязкости.

Следует отметить, что предложенная нами гидродинамическая модель (2) хотя и существенно проще, чем исходная система уравнений Навье-Стокса, тем не менее является весьма содержательной. Как будет установлено ниже, при согласованном стремлении параметров $\varepsilon$ и $\nu$ к нулю в ней происходит развитие турбулентности по Ландау, а при $\varepsilon \sim 1, \nu \rightarrow 0$ реализуется сценарий Ландау-Селла.

\section{2. РАЗРЕШИМОСТЬ НАЧАЛЬНО-КРАЕВОЙ ЗАДАЧИ}

Краевую задачу (2) будем рассматривать как динамическую систему в фазовом пространстве $\stackrel{\circ}{W} \underset{2}{1}(0,1) \times L_{2}(0,1)$, где, как обычно, через $\stackrel{\circ}{W} \underset{2}{2}(0,1)$ обозначено замыкание по норме

$$
\|u\|_{W_{2}^{1}}=\left(\int_{0}^{1} u^{2} d x+a^{2} \int_{0}^{1}\left(u^{\prime}\right)^{2} d x\right)^{1 / 2}
$$

линеала гладких функций $u(x), 0 \leqslant x \leqslant 1$, удовлетворяющих граничным условиям $u(0)=u(1)=0$. Для того чтобы определить понятие обобщенного решения этой задачи с начальными условиями из указанного фазового пространства, положим $w_{1}=\mathcal{D} u, w_{2}=u_{t}$, где $\mathcal{D}: \stackrel{\circ}{W_{2}^{1}}(0,1) \rightarrow L_{2}(0,1)$ - арифметический квадратный корень из оператора $I-a^{2} d^{2} / d x^{2}, I-$ единичный оператор. В итоге в пространстве $E=$ $L_{2}\left([0,1] ; \mathbb{R}^{2}\right)$, состоящем из вектор-функций

$$
w=\left(w_{1}, w_{2}\right)^{\mathrm{T}}, \quad w_{1}, w_{2} \in L_{2}(0,1), \quad\|w\|_{E}=\left(\int_{0}^{1}\left(w_{1}^{2}+w_{2}^{2}\right) d x\right)^{1 / 2},
$$

получаем абстрактное дифференциальное уравнение вида

$$
\dot{w}=A w+F(w)
$$

Здесь $A=\mathcal{V}_{0} \mathcal{D}-\mathcal{V}_{1} \mathcal{D}^{2}+\mathcal{V}_{2}$,

$$
\begin{gathered}
\mathcal{V}_{0}=\left(\begin{array}{rr}
0 & 1 \\
-1 & 0
\end{array}\right), \quad \mathcal{V}_{1}=\left(\begin{array}{cc}
0 & 0 \\
0 & \nu / a^{2}
\end{array}\right), \quad \mathcal{V}_{2}=\left(\begin{array}{cc}
0 & 0 \\
0 & \varepsilon+\nu / a^{2}
\end{array}\right), \\
F(w)=\left(0,-b\left(\mathcal{D}^{-1} w_{1}\right)^{5}-w_{2} \int_{0}^{1} w_{2}^{2} d x\right)^{\mathrm{T}},
\end{gathered}
$$

а операторы $\mathcal{D}$ и $\mathcal{D}^{2}$ применяются к вектор-функции (6) покомпонентно. Например, $\mathcal{D} w=\left(\mathcal{D} w_{1}, \mathcal{D} w_{2}\right)^{\mathrm{T}}$.

Убедимся, что оператор $A$ порождает в $E$ сильно непрерывную по $t \geqslant 0$ полугруппу линейных ограниченных операторов $\exp (A t)$. Для этого обратимся к разложениям Фурье:

$$
\begin{gathered}
w=\sum_{k=1}^{\infty} w_{(k)} e_{k}(x), \quad \mathcal{D} w=\sum_{k=1}^{\infty} \omega_{k} w_{(k)} e_{k}(x), \quad w_{(k)}=\int_{0}^{1} w e_{k}(x) d x, \\
e_{k}(x)=\sqrt{2} \sin (k \pi x), \quad \omega_{k}=\sqrt{1+a^{2} \pi^{2} k^{2}}, \quad k \geqslant 1 .
\end{gathered}
$$


Из представлений (9) и из явного выражения для $A$ очевидным образом следует, что интересующая нас полугруппа действует на любой элемент $w \in E$ по правилу

$$
\exp (A t) w=\sum_{k=1}^{\infty} \exp \left[\left(\omega_{k} \mathcal{V}_{0}-\omega_{k}^{2} \mathcal{V}_{1}+\mathcal{V}_{2}\right) t\right] w_{(k)} e_{k}(x)
$$

Наличие полугруппы (10) позволяет определить понятие обобщенного решения в первую очередь для уравнения (7): под обобщенным решением этого уравнения с произвольным начальным условием $w(0)=w_{0} \in E$ будем понимать непрерывную вектор-функцию $w(t)$ со значениями в $E$, удовлетворяющую на некотором отрезке $0 \leqslant t \leqslant \bar{t}$ интегральному уравнению

$$
w(t)=\exp (A t) w_{0}+\int_{0}^{t} \exp (A(t-s)) F(w(s)) d s .
$$

Полагая затем $u(t, \cdot)=\mathcal{D}^{-1} w_{1}(t), u_{t}(t, \cdot)=w_{2}(t)$, где $w_{1}(t), w_{2}(t)$ - компоненты введенной в (6) вектор-функции $w(t)$, получаем искомое обобщенное решение

$$
\left(u(t, x), u_{t}(t, x)\right) \in \stackrel{\circ}{W} \underset{2}{1}(0,1) \times L_{2}(0,1)
$$

исходной краевой задачи (2). Подчеркнем, что включение в (12) имеет место по переменной $x$ при любом фиксированном $t \in[0, \bar{t}]$. При этом сама вектор-функция $\left(u, u_{t}\right)$ непрерывна по $t$ в пространстве $\stackrel{\circ}{W} \underset{2}{1}(0,1) \times L_{2}(0,1)$ с нормой

$$
\left\|\left(u, u_{t}\right)\right\|_{\stackrel{\circ}{2}_{2}^{1} \times L_{2}}=\left(\|u\|_{W_{2}^{1}}^{2}+\left\|u_{t}\right\|_{L_{2}}^{2}\right)^{1 / 2} .
$$

Обратимся теперь к вопросу о существовании обобщенных решений. Справедливо следующее утверждение.

Теорема 1. Обобщенное решение (12) краевой задачи (2) с произвольными начальными условиями

$$
\left.u\right|_{t=0}=u_{0}(x) \in \stackrel{\circ}{\stackrel{W}{W}_{2}^{1}}(0,1),\left.\quad u_{t}\right|_{t=0}=u_{1}(x) \in L_{2}(0,1)
$$

существует и единственно при всех $t \geqslant 0$.

При обосновании сформулированной теоремы нам потребуются некоторые факты из теории так называемых абстрактных полулинейных дифференциальных уравнений. В связи с этим будем рассматривать уравнение (7) в произвольном вещественном банаховом пространстве $E$ с нормой $\|\cdot\|$, предполагая, что, во-первых, $A: E_{A} \rightarrow E-$ замкнутый линейный оператор с плотной в $E$ областью определения $E_{A}$, являющийся инфинитезимальным производящим для $C^{0}$-полугруппы линейных ограниченных операторов $\exp (A t), t \geqslant 0$; во-вторых, нелинейный оператор $F: E \rightarrow E$ бесконечно дифференцируем по Фреше и ограничен на любом ограниченном подмножестве $\Omega \subset E$ вместе с любым конечным числом своих производных. Подчеркнем, что перечисленным свойствам заведомо удовлетворяет нелинейный оператор $F$ из (8). Уравнение (7) при выполнении указанных условий будем называть полулинейным. 
Дополним уравнение (7) начальным условием

$$
w(0)=w_{0},
$$

где $w_{0}$ - произвольный элемент из $E$. Под обобщенным решением задачи Коши (7), (15), как уже было сказано выше, понимается непрерывное решение соответствующего интегрального уравнения (11). Пусть теперь $w_{0} \in E_{A}$. Тогда классическим решением задачи Коши (7), (15) назовем такую непрерывно дифференцируемую на некотором отрезке $0 \leqslant t \leqslant \bar{t}$ вектор-функцию $w=w(t)$, что, во-первых, $w(t) \in E_{A}$ и $A w(t)$ непрерывна при каждом $t \in[0, \bar{t}]$; во-вторых, функция $w(t)$ на рассматриваемом отрезке удовлетворяет уравнению (7), а при $t=0$ - начальному условию (15).

Общая теория абстрактных полулинейных дифференциальных уравнений содержится, например, в монографиях [10], [11]. Из приведенных там построений вытекает следующая серия утверждений.

ЛЕмма 1. По любому ограниченному множеству $\Omega \subset$ Е можно указать такое $t_{0}=t_{0}(\Omega)>0$, что каждое обобщенное решение $w=w\left(t, w_{0}\right)$ уравнения $(7)$ с начальным условием $w\left(0, w_{0}\right)=w_{0} \in \Omega$ однозначно определено на отрезке $0 \leqslant t \leqslant t_{0}$. $B$ случае $w_{0} \in \Omega \cap E_{A}$ это решение при всех $t \in\left[0, t_{0}\right]$ будет классическим.

ЛЕмма 2. Пусть задано произвольное (вообще говоря, обобщенное) решение $w=$ $w_{*}(t)$ уравнения $(7)$ на некотором отрезке $0 \leqslant t \leqslant \bar{t}$. Тогда найдется такое достаточно малое $r_{0}>0$, что любое обобщенное решение $w=w\left(t, w_{0}\right)$ этого уравнения с начальным условием $w\left(0, w_{0}\right)=w_{0} \in B, B=\left\{w \in E:\left\|w-w_{*}(0)\right\| \leqslant r_{0}\right\}$, существует на отрезке $0 \leqslant t \leqslant \bar{t} u$ непрерывно по совокупности переменных $\left(t, w_{0}\right) \in[0, \bar{t}] \times B$. Если же дополнительно $w_{0} \in E_{A}$, то соответствующее решение $w\left(t, w_{0}\right)$ при всех $t \in[0, \bar{t}]$ оказывается классическим.

ЛЕмма 3. Для любого обобщенного решения $w=w_{*}(t)$, определенного на отрезке $0 \leqslant t \leqslant \bar{t}$, найдется такая последовательность классических решений $w=w_{n}(t)$, $t \in[0, \bar{t}], n \geqslant 1$, иmo

$$
\lim _{n \rightarrow \infty} \max _{0 \leqslant t \leqslant \bar{t}}\left\|w_{*}(t)-w_{n}(t)\right\|=0 .
$$

Скажем несколько слов об обосновании каждой из приведенных лемм. Существование и единственность обобщенных решений, о которых говорится в лемме 1, вытекает из анализа интегрального уравнения (11). Действительно, обозначим через П оператор, порождаемый правой частью этого уравнения в пространстве $C\left(\left[0, t_{0}\right] ; E\right)$ непрерывных на отрезке $0 \leqslant t \leqslant t_{0}$ вектор-функций $w(t) \in E$ с нормой $\|w(t)\|_{C}=$ $\max _{0 \leqslant t \leqslant t_{0}}\|w(t)\|$. Опираясь на свойства ограниченности и гладкости оператора $F$ и используя для оператора $\exp (A t)$ известную оценку $\|\exp (A t)\|_{E \rightarrow E} \leqslant c_{1} e^{c_{2} t}, t \geqslant 0$, $c_{1}=$ const $>0, c_{2}=$ const $\in \mathbb{R}$, нетрудно установить существование для любого фиксированного $r_{0}>0$ такого не зависящего от $w_{0} \in \Omega$ достаточно малого значения $t_{0}=t_{0}\left(r_{0}, \Omega\right)>0$, что оператор $\Pi$ преобразует в себя множество $\left\{w(t) \in C\left(\left[0, t_{0}\right] ; E\right):\left\|w(t)-\exp (A t) w_{0}\right\|_{C} \leqslant r_{0}\right\}$ и оказывается сжимающим. Отсюда очевидным образом следует, что любое обобщенное решение $w\left(t, w_{0}\right)$ уравнения $(7)$ с начальным условием $w_{0} \in \Omega$ однозначно определено на отрезке $0 \leqslant t \leqslant t_{0}$. Более 
того, используя введенные в работе [11] (см. с. 71-76) конструкции, можно показать, что при $w_{0} \in \Omega \cap E_{A}$ это решение будет классическим.

Доказательство существования обобщенных решений из леммы 2 связано с анализом аналогичного (11) интегрального уравнения

$$
\Pi\left(h, w_{0}\right)=0, \quad\left(h, w_{0}\right) \in C([0, \bar{t}] ; E) \times E,
$$

где $h(t)=w\left(t, w_{0}\right)-w_{*}(t)$,

$$
\begin{aligned}
\Pi\left(h, w_{0}\right)= & h(t)-\exp (A t)\left(w_{0}-w_{*}(0)\right)- \\
& -\int_{0}^{t} \exp (A(t-s))\left[F\left(w_{*}(s)+h(s)\right)-F\left(w_{*}(s)\right)\right] d s .
\end{aligned}
$$

Непосредственная проверка показывает, что в точке $h=0, w_{0}=w_{*}(0)$ к этому уравнению применима теорема о неявном отображении по переменной $h$. Таким образом, обобщенные решения уравнения (7), о которых говорится в лемме 2, задаются равенством $w\left(t, w_{0}\right)=w_{*}(t)+h\left(t, w_{0}\right)$, где $h\left(t, w_{0}\right) \in C([0, \bar{t}] ; E)-$ решение уравнения (17), существующее при всех $w_{0}$, близких к $w_{*}(0)$, и непрерывное по совокупности переменных.

Для обоснования утверждения леммы 2, касающегося классических решений, достаточно показать, что любое обобщенное решение $w(t)$ уравнения $(7)$, определенное на произвольном отрезке $t \in[0, \bar{t}]$, в случае $w(0) \in E_{A}$ является классическим. Проверка этого факта базируется на лемме 1. В самом деле, взяв в условиях этой леммы в качестве начального множества $\Omega$ кривую $\{w=w(t), 0 \leqslant t \leqslant \bar{t}\}$, последовательно убеждаемся, что интересующее нас решение будет классическим на отрезках $\left[0, t_{0}\right]$, $\left[t_{0}, 2 t_{0}\right],\left[2 t_{0}, 3 t_{0}\right]$ и т.д., где $t_{0}=t_{0}(\Omega)>0$.

Лемма 3 непосредственно вытекает из леммы 2. Для того чтобы убедиться в этом, возьмем такую последовательность $w_{n} \in E_{A}$ (существующую в силу плотности $E_{A}$ в $E)$, что $\left\|w_{n}-w_{*}(0)\right\| \rightarrow 0$ при $n \rightarrow \infty$. Далее, без ограничения общности будем считать, что $w_{n} \in E_{A} \cap B$, где $B=\left\{w \in E:\left\|w-w_{*}(0)\right\| \leqslant r_{0}\right\}$, а постоянная $r_{0}>0$ выбрана в соответствии с леммой 2 по решению $w=w_{*}(t)$. В этом случае каждое решение $w_{n}(t)=w\left(t, w_{n}\right)$ уравнения (7) с начальным условием $w\left(0, w_{n}\right)=w_{n}$ заведомо является классическим и определено на всем отрезке $0 \leqslant t \leqslant \bar{t}$. Что же касается требуемого предельного равенства (16), то оно является очевидным следствием непрерывности функции $w\left(t, w_{0}\right)$ по совокупности переменных $\left(t, w_{0}\right) \in$ $[0, \bar{t}] \times B$.

В дальнейшем нам потребуется еще одна лемма, связанная с продолжением решений уравнения (7). Прежде чем ее формулировать, для каждого решения $w(t)$ этого уравнения с начальным условием (15) определим так называемый максимальный полуинтервал существования $\left[0, t_{\max }\right)$, где

$$
t_{\max }=\sup \left\{t_{0}: w(t) \text { существует на отрезке } t \in\left[0, t_{0}\right]\right\} .
$$

В силу леммы 1 множество значений $t_{0}$, по которым берется точная верхняя грань в (18), заведомо не пусто. Ясно также, что любое решение $w(t)$ однозначно продолжается на свой максимальный полуинтервал $\left[0, t_{\max }\right)$, причем не исключен случай 
$t_{\max }=+\infty$. Если же, напротив, $t_{\max }<+\infty$, то оказывается справедливым следующее утверждение.

ЛЕмма 4. Предположим, что для некоторого обобщенного решения $w(t)$ уравнения (7) конечна величина (18). Тогда имеет место предельное равенство

$$
\lim _{t \rightarrow t_{\max }-0}\|w(t)\|=+\infty .
$$

ДоКАЗАТЕЛЬСТво леммы проводится от противного. Действительно, предположим, что соотношение (19) не выполняется. В этом случае существуют такая последовательность моментов времени $t_{n}, t_{n} \rightarrow t_{\max }-0$ при $n \rightarrow \infty$, и такая постоянная $M>0$, что $\left\|w\left(t_{n}\right)\right\| \leqslant M, n \geqslant 1$. Далее, в условиях леммы 1 в качестве ограниченного множества $\Omega$ возьмем $\left\{w\left(t_{n}\right), n \geqslant 1\right\}$. Тогда, очевидно, решение $w(t)$ будет определено на всех отрезках вида $\left[t_{n}, t_{n}+t_{0}\right], n \geqslant 1$, где $t_{0}=t_{0}(\Omega)>0$. Ясно также, что при достаточно больших $n$ выполняется включение $t_{\max } \in\left(t_{n}, t_{n}+t_{0}\right)$. Но это противоречит определению $t_{\max }$ (см. (18)). Лемма доказана.

Обратимся теперь непосредственно к доказательству теоремы 1. В связи с этим фиксируем произвольно начальные функции (14), а затем перейдем в начальнокраевой задаче (2), (14) к переменным (6). В результате получим задачу Коши (7), $(15)$, в которой $w_{0}=\left(w_{1}^{0}, w_{2}^{0}\right)^{\mathrm{T}}, w_{1}^{0}=\mathcal{D} u_{0}(x), w_{2}^{0}=u_{1}(x)$. Применяя далее к упомянутой задаче лемму 1 , убеждаемся, что ее обобщенное решение $w(t)=\left(w_{1}(t), w_{2}(t)\right)^{\mathrm{T}}$ однозначно определено на своем максимальном полуинтервале существования $\left[0, t_{\max }\right)$. Наконец, осуществляя обратный переход от переменных $(6)$ к исходным переменным $\left(u, u_{t}\right)$, получаем единственное обобщенное решение

$$
\left(u(t, x), u_{t}(t, x)\right) \in \stackrel{\circ}{W_{2}^{1}}(0,1) \times L_{2}(0,1), \quad t \in\left[0, t_{\max }\right),
$$

интересующей нас смешанной задачи (2), (14).

Итак, для завершения обоснования теоремы 1 осталось лишь установить, что фигурирующая в $(20)$ величина $t_{\text {max }}$ равна $+\infty$. В силу леммы 4 для этого достаточно показать справедливость для любого $t \in\left[0, t_{\max }\right)$ априорной оценки вида

$$
\left\|\left(u, u_{t}\right)\right\|_{W_{2}^{1} \times L_{2}} \leqslant c \cdot e^{\varepsilon t}, \quad c=\text { const }>0,
$$

препятствующей “уходу” решения $\left(u, u_{t}\right)$ в бесконечность за конечное время.

Для того чтобы убедиться в выполнении требуемого неравенства, фиксируем произвольно значение $\bar{t} \in\left[0, t_{\max }\right)$ и на отрезке $0 \leqslant t \leqslant \bar{t}$ аппроксимируем построенное выше обобщенное решение (20) классическими решениями. Согласно лемме 3 найдется такая последовательность классических решений $u_{n}(t, x), n \geqslant 1$, краевой задачи (2), что

$$
\max _{0 \leqslant t \leqslant \bar{t}}\left\|u-u_{n}\right\|_{\mathscr{O}_{2}^{1}} \rightarrow 0, \quad \max _{0 \leqslant t \leqslant \bar{t}}\left\|\frac{\partial u}{\partial t}-\frac{\partial u_{n}}{\partial t}\right\|_{L_{2}} \rightarrow 0, \quad n \rightarrow \infty .
$$

Остановимся на некоторых необходимых в дальнейшем свойствах функций $u_{n}(t, x)$. Нетрудно увидеть, что область определения оператора $A$ (см. $\left.(7),(8)\right)$ задается равенством

$$
E_{A}=\left\{w=\left(w_{1}, w_{2}\right)^{\mathrm{T}} \in E: w_{1} \in \stackrel{\circ}{W_{2}^{1}}(0,1), w_{2} \in \stackrel{\circ}{W}_{2}^{2}(0,1)\right\} .
$$


Отсюда и из приведенного выше общего определения классических решений полулинейных уравнений вида (7) вытекает, что в данном случае, во-первых,

$$
\begin{aligned}
u_{n}(t, \cdot) & \in C^{1}\left([0, \bar{t}] ; \stackrel{\circ}{W}{ }_{2}^{1}\right) \cap C\left([0, \bar{t}] ; \stackrel{\circ}{W_{2}^{2}}\right), \\
\frac{\partial u_{n}}{\partial t}(t, \cdot) & \in C^{1}\left([0, \bar{t}] ; \stackrel{\circ}{L}_{2}\right) \cap C\left([0, \bar{t}] ; \stackrel{\circ}{W_{2}^{2}}\right) ;
\end{aligned}
$$

во-вторых, при любом фиксированном $t \in[0, \bar{t}]$ и при каждом $n \geqslant 1$

$$
\frac{\partial^{2} u_{n}}{\partial t^{2}}-a^{2} \frac{\partial^{2} u_{n}}{\partial x^{2}}+u_{n}+b u_{n}^{5}=\varepsilon \frac{\partial u_{n}}{\partial t}+\nu \frac{\partial^{3} u_{n}}{\partial x^{2} \partial t}-\frac{\partial u_{n}}{\partial t} \int_{0}^{1}\left(\frac{\partial u_{n}}{\partial t}\right)^{2} d x .
$$

Точнее говоря, в силу (24) все фигурирующие в (25) производные заведомо принадлежат пространству $L_{2}(0,1)$ по $x$, и само соотношение $(25)$ понимается в смысле равенства в $L_{2}(0,1)$ по переменной $x$.

Покажем сначала, что интересующая нас оценка (21) выполняется для решений $u_{n}(t, x), n \geqslant 1$. С этой целью умножим левую и правую части соотношения (25) на $\partial u_{n} / \partial t$ и проинтегрируем по $x$ в пределах от 0 до 1 . В результате убеждаемся, что

$$
\begin{aligned}
\frac{1}{2} \frac{d}{d t} \ell\left(u_{n}, \frac{\partial u_{n}}{\partial t}\right)= & \varepsilon \int_{0}^{1}\left(\frac{\partial u_{n}}{\partial t}\right)^{2} d x-\nu \int_{0}^{1}\left(\frac{\partial^{2} u_{n}}{\partial x \partial t}\right)^{2} d x- \\
& -\left(\int_{0}^{1}\left(\frac{\partial u_{n}}{\partial t}\right)^{2} d x\right)^{2} \leqslant \varepsilon \int_{0}^{1}\left(\frac{\partial u_{n}}{\partial t}\right)^{2} d x
\end{aligned}
$$

и, соответственно,

$$
\ell\left(u_{n}, \frac{\partial u_{n}}{\partial t}\right) \leqslant\left.\ell\left(u_{n}, \frac{\partial u_{n}}{\partial t}\right)\right|_{t=0}+2 \varepsilon \int_{0}^{t} \varkappa_{n}(\tau) d \tau
$$

где $\varkappa_{n}(t)=\int_{0}^{1}\left(\partial u_{n} / \partial t\right)^{2} d x, \ell\left(u, u_{t}\right)$ - функционал, определяемый левой частью формулы (4). Далее, учитывая вытекающие из (4), (5), (13) очевидные неравенства

$$
\varkappa_{n}(t) \leqslant \ell\left(u_{n}, \frac{\partial u_{n}}{\partial t}\right), \quad\left\|\left(u_{n}, \frac{\partial u_{n}}{\partial t}\right)\right\|_{W_{2}^{1} \times L_{2}}^{2} \leqslant \ell\left(u_{n}, \frac{\partial u_{n}}{\partial t}\right),
$$

из неравенства (26) и леммы Гронуолла-Беллмана последовательно получаем следующие оценки:

$$
\varkappa_{n}(t) \leqslant\left.\ell\left(u_{n}, \frac{\partial u_{n}}{\partial t}\right)\right|_{t=0} \cdot e^{2 \varepsilon t}, \quad \ell\left(u_{n}, \frac{\partial u_{n}}{\partial t}\right) \leqslant\left.\ell\left(u_{n}, \frac{\partial u_{n}}{\partial t}\right)\right|_{t=0} \cdot e^{2 \varepsilon t} .
$$

Отсюда, в свою очередь, следует искомая оценка для классических решений $u_{n}(t, x)$ : при любом $t \in[0, \bar{t}]$

$$
\left\|\left(u_{n}, \frac{\partial u_{n}}{\partial t}\right)\right\|_{\stackrel{\circ}{2}_{2}^{1} \times L_{2}} \leqslant \sqrt{\left.\ell\left(u_{n}, \frac{\partial u_{n}}{\partial t}\right)\right|_{t=0}} \cdot e^{\varepsilon t} .
$$


На завершающем этапе, привлекая свойства (22), перейдем в (27) к пределу при $n \rightarrow \infty$. В итоге на отрезке $0 \leqslant t \leqslant \bar{t}$ получаем требуемую априорную оценку (21) с константой $c=\sqrt{\ell\left(u_{0}, u_{1}\right)}$, где $u_{0}, u_{1}$ - начальные функции из (14). Остается заметить, что в силу произвольности $\bar{t} \in\left(0, t_{\max }\right)$ эта оценка справедлива на всем полуинтервале $\left[0, t_{\max }\right)$. Теорема 1 доказана.

Следует добавить, что утверждение теоремы 1 сохраняется (вместе с обоснованием) и в случае $\nu=0$. В некоторой корректировке нуждаются только равенство (23) и свойства (24) классических решений: при $\nu=0$ в (23), (24) для компонент $w_{2}$ и $\partial u_{n} / \partial t$ должны выполняться включения

$$
w_{2} \in \stackrel{\circ}{W_{2}^{1}}(0,1), \quad \frac{\partial u_{n}}{\partial t}(t, \cdot) \in C^{1}\left([0, \bar{t}] ; L_{2}\right) \cap C\left([0, \bar{t}] ; \stackrel{\circ}{W}_{2}^{1}\right) .
$$

\section{3. АНАЛИЗ ЛОКАЛЬНЫХ АТТРАКТОРОВ}

В данном разделе мы изучим локальные (располагающиеся в некоторой достаточно малой окрестности нулевого состояния равновесия) аттракторы краевой задачи (2) при условии малости параметров $\varepsilon$ и $\nu$. В связи с этим рассмотрим краевую задачу

$$
\begin{gathered}
u_{t t}-a^{2} u_{x x}+u=\varepsilon \frac{\partial}{\partial t}\left(u+\nu_{0} u_{x x}\right)-\varepsilon u_{t} \int_{0}^{1}\left(u_{t}\right)^{2} d x-\varepsilon^{2} b u^{5}, \\
\left.u\right|_{x=0}=\left.u\right|_{x=1}=0,
\end{gathered}
$$

получающуюся из $(2)$ при $0<\varepsilon \ll 1, \nu=\nu_{0} \varepsilon, \nu_{0}=$ const $\geqslant 0$ после замены $u / \sqrt{\varepsilon} \rightarrow u$.

Для исследования аттракторов задачи (28) воспользуемся бесконечномерным аналогом известного асимптотического метода Крылова-Боголюбова-Митропольского [12]. Суть упомянутого метода состоит в том, что возможные автоколебательные режимы рассматриваемой задачи ищутся в виде формального разложения

$$
u=u_{0}(t, \tau, x)+\varepsilon u_{1}(t, \tau, x)+\cdots, \quad \tau=\varepsilon t .
$$

Здесь

$$
\begin{aligned}
& u_{j}(t, \tau, x)=\sum_{n=1}^{\infty} u_{n, j}(t, \tau) e_{n}(x), \quad j=0,1, \\
& u_{n, 0}=z_{n}(\tau) e^{i \omega_{n} t}+\bar{z}_{n}(\tau) e^{-i \omega_{n} t}, \quad n \geqslant 1,
\end{aligned}
$$

функции $e_{n}(x)$ и часто́ты $\omega_{n}$ те же, что и в $(9)$, а неизвестные (подлежащие определению) комплексные амплитуды $z_{n}(\tau), n \geqslant 1$, таковы, что сходится ряд с общим членом $\omega_{n}^{2}\left|z_{n}\right|^{2}$ (в этом случае после подстановки соотношений (31) в ряд (30) при $j=0$ получается функция из класса $\stackrel{\circ}{W} \underset{2}{1}$ по переменной $x)$. Что касается поправки порядка $\varepsilon$ из $(29)$, то фигурирующие в ней функции $u_{n, 1}(t, \tau)$ будем искать в классе формальных тригонометрических рядов переменных $\omega_{k} t, k \geqslant 1$.

Итак, подставим в краевую задачу (28) разложения (29), (30) и приравняем коэффициенты при $\varepsilon$. В результате приходим к счетной системе линейных неоднородных 
дифференциальных уравнений

$$
\ddot{u}_{n, 1}+\omega_{n}^{2} u_{n, 1}=f_{n}(t, \tau), \quad n \geqslant 1,
$$

где, как обычно, точкой обозначена производная по $t$, переменная $\tau$ считается параметром, а функции $f_{n}(t, \tau)$ имеют вид

$$
\begin{aligned}
& f_{n}=f_{n}^{0} e^{i \omega_{n} t}+\overline{f_{n}^{0}} e^{-i \omega_{n} t}-\dot{u}_{n, 0} \sum_{k=1}^{\infty}\left(\dot{u}_{k, 0}\right)^{2}, \\
& f_{n}^{0}=i \omega_{n}\left(1-\nu_{0} \pi^{2} n^{2}\right) z_{n}-2 i \omega_{n} \frac{d z_{n}}{d \tau} .
\end{aligned}
$$

Необходимым и достаточным условием разрешимости уравнений (32) в интересующем нас классе функций является отсутствие в их правых частях (33) гармоник $e^{ \pm i \omega_{n} t}$. Добиться равенства нулю коэффициентов при указанных гармониках удается за счет имеющихся в запасе комплексных амплитуд $z_{n}$, для которых при этом получается некоторая счетная система обыкновенных дифференциальных уравнений. Нетрудно проверить, что после перехода к переменным $\xi_{n}=\omega_{n} z_{n}, n \geqslant 1$, упомянутая система записывается в виде

$$
2 \frac{d \xi_{n}}{d \tau}=\left[1-\nu_{0} \pi^{2} n^{2}-3\left|\xi_{n}\right|^{2}-2 \sum_{\substack{k \geqslant 1, k \neq n}}\left|\xi_{k}\right|^{2}\right] \xi_{n}, \quad n \geqslant 1
$$

Завершая описание алгоритмической части нашего метода, введем в рассмотрение пространство последовательностей $Z_{0}$ (над полем вещественных чисел), состоящее из бесконечномерных векторов

$$
\begin{gathered}
\xi=\left(\xi_{1}, \bar{\xi}_{1}, \ldots, \xi_{n}, \bar{\xi}_{n}, \ldots\right), \quad \xi_{k} \in \mathbb{C}, \quad k \geqslant 1, \\
\|\xi\|=\left(\sum_{k=1}^{\infty}\left|\xi_{k}\right|^{2}\right)^{1 / 2}<\infty .
\end{gathered}
$$

Заметим, что согласно работе [10] система (34) порождает абстрактное полулинейное параболическое уравнение в пространстве $Z_{0}$. Опираясь на известные свойства таких уравнений (см. леммы 1-4) и очевидные априорные оценки $\left|\xi_{n}(\tau)\right|^{2} \leqslant\left|\xi_{n}(0)\right|^{2} e^{\tau}$, $n \geqslant 1, \tau \geqslant 0$, нетрудно убедиться, что любое решение $\xi(\tau)=\left(\xi_{1}(\tau), \bar{\xi}_{1}(\tau), \ldots, \xi_{n}(\tau)\right.$, $\bar{\xi}_{n}(\tau), \ldots$ ) данной системы с начальным условием $\xi(0) \in Z_{0}$ определено на полуоси $\tau \geqslant 0$. Поэтому если мы зафиксируем какое-либо из таких решений и подставим $z_{n}(\tau)=\xi_{n}(\tau) / \omega_{n}, n \geqslant 1$, в (33), то все уравнения (32) станут разрешимыми в классе тригонометрических рядов, а их решения примут вид

$$
u_{n, 1}=-\frac{i}{8 \omega_{n}^{2}}\left(\xi_{n}^{3} e^{3 i \omega_{n} t}-\overline{\xi_{n}^{3}} e^{-3 i \omega_{n} t}\right)+i \xi_{n} u_{n, 1}^{0}-i \bar{\xi}_{n} \overline{u_{n, 1}^{0}},
$$

где

$$
u_{n, 1}^{0}=\sum_{\substack{k \geqslant 1 \\ k \neq n}} \frac{1}{4 \omega_{k}}\left(\frac{\overline{\xi_{k}^{2}}}{\omega_{n}-\omega_{k}} e^{i\left(\omega_{n}-2 \omega_{k}\right) t}-\frac{\xi_{k}^{2}}{\omega_{n}+\omega_{k}} e^{i\left(\omega_{n}+2 \omega_{k}\right) t}\right) .
$$


Чтобы придать изложенным формальным построениям необходимую строгость, перейдем от (34) к системе для амплитуд $\rho_{n}=\left|\xi_{n}\right|^{2}$ :

$$
\frac{d \rho_{n}}{d \tau}=\left[1-\nu_{0} \pi^{2} n^{2}-3 \rho_{n}-2 \sum_{\substack{k \geqslant 1, k \neq n}} \rho_{k}\right] \rho_{n}, \quad n \geqslant 1,
$$

и будем рассматривать последнюю как самостоятельную динамическую систему в конусе $K$ векторов с неотрицательными координатами из пространства

$$
l=\left\{\rho=\left(\rho_{1}, \ldots, \rho_{n}, \ldots\right):\|\rho\|=\sum_{n=1}^{\infty}\left|\rho_{n}\right|<\infty\right\} .
$$

Предположим, что система (36) имеет состояние равновесия с конечным числом ненулевых координат $\rho_{n_{j}}=\rho_{n_{j}}^{0}>0, j=1, \ldots, k$, экспоненциально устойчивое или дихотомичное (в метрике фазового пространства (37)). Тогда справедливо следующее утверждение.

ТЕОрема 2. Введенному выше состоянию равновесия системы (36) в исходной краевой задаче (28) при всех $\varepsilon \in\left(0, \varepsilon_{0}\right]$, где $\varepsilon_{0}>0$ достаточно мало, соответствует $k$-мерный инвариантный тор $T_{k}$ с теми же свойствами устойчивости. Указанный тор задается уравнениями

$$
\begin{aligned}
u & =\sum_{j=1}^{k} \frac{2}{\omega_{n_{j}}} \sqrt{\rho_{n_{j}}^{0}} \cos \varphi_{j} \cdot e_{n_{j}}(x)+\varepsilon U_{*}(\varphi, x, \varepsilon), \\
\frac{d \varphi}{d t} & =\omega+\varepsilon^{2} \Phi_{*}(\varphi, \varepsilon),
\end{aligned}
$$

где $\varphi=\left(\varphi_{1}, \ldots, \varphi_{k}\right)^{\mathrm{T}}, \omega=\left(\omega_{n_{1}}, \ldots, \omega_{n_{k}}\right)^{\mathrm{T}}$, а $2 \pi$-периодические по $\varphi$ функции $U_{*}, \Phi_{*}$ ограничены равномерно по $\varphi, \varepsilon$ (вместе с любым биксированным числом производных по ч) в метриках пространств $\stackrel{\circ}{W} \underset{2}{1}(0,1)$ u $\mathbb{R}^{k}$ соответственно.

Сформулированная теорема представляет собой частный случай установленного в монографии [11] (см. гл. 1) общего результата о бифуркации инвариантных торов в абстрактных нелинейных системах. Отметим, что в [11] реализован геометрический подход к проблеме существования и устойчивости инвариантных торов, основанный на исследовании динамических свойств оператора сдвига по траекториям рассматриваемой системы за фиксированное конечное время $t_{0}>0$. Что касается краевой задачи (28), то для нее ввести такого рода оператор позволяет полученная выше теорема 1.

Теорема 2 сводит интересующую нас проблему аттракторов краевой задачи (28) к поиску устойчивых состояний равновесия системы (36). Для формулировки соответствующего результата рассмотрим монотонно убывающую последовательность

$$
\bar{\nu}_{N}=\frac{3}{\pi^{2} N\left(4 N^{2}-1\right)}, \quad N \geqslant 1
$$

критических значений параметра $\nu_{0}$. Динамику системы (36) по этому параметру описывает следующее утверждение. 
ТЕорема 3. Предположим, что параметр $\nu_{0}$ удовлетворяет неравенствам

$$
\bar{\nu}_{N+1}<\nu_{0}<\bar{\nu}_{N}
$$

при некотором натуральном $N$. Тогда система (36) имеет экспоненииально устойчивое состояние равновесия $O_{N}$ с координатами

$$
\rho_{n}=\left\{\begin{array}{lll}
\frac{1}{2 N+1}+\frac{\nu_{0} \pi^{2}}{3} N(N+1)-\nu_{0} \pi^{2} n^{2} & \text { npu } & n=1, \ldots, N \\
0 & \text { npu } & n \geqslant N+1 .
\end{array}\right.
$$

Все остальные возможные состояния равновесия этой системы, лежащие в конусе $K$, при условиях (40) экспоненциалъно неустойчивы.

ДокаЗАтеЛьство. Факт существования у системы $(36)$ при $\nu_{0} \in\left[0, \bar{\nu}_{N}\right)$ состояния равновесия $O_{N}$ проверяется непосредственно. Система в вариациях на нем распадается на систему из $N$ уравнений

$$
\dot{h}=-B h, \quad h=\left(h_{1}, \ldots, h_{N}\right)^{\mathrm{T}},
$$

где элементы $b_{j, r}, j, r=1, \ldots, N$, матрицы $B$ таковы, что $b_{j, j}=3 \rho_{j}, b_{j, r}=2 \rho_{j}$ при $j \neq r$, и на серию скалярных уравнений

$$
\dot{h}_{m}=\left(1-2 S-\nu_{0} \pi^{2} m^{2}\right) h_{m}, \quad S=\sum_{j=1}^{N} \rho_{j}, \quad m \geqslant N+1 .
$$

Анализ свойств устойчивости системы (42) сводится, очевидно, к исследованию расположения корней уравнения $\operatorname{det}(\lambda I+B)=0$, здесь $I$ - единичная матрица. Покажем, что все они вещественны и отрицательны.

Для того чтобы раскрыть введенный выше определитель, сначала вычтем последний столбец матрицы $\lambda I+B$ из всех предыдущих. После этого добавим к последней строке получившейся матрицы все остальные ее строки, умножив $j$-ю строку на $\left(\rho_{N}+\lambda\right) /\left(\rho_{j}+\lambda\right), j=1, \ldots, N-1$. В итоге интересующее нас уравнение $\operatorname{det}(\lambda I+B)=0$ преобразуется к виду

$$
\left(\rho_{1}+\lambda\right) \ldots\left(\rho_{N}+\lambda\right)\left[1+\sum_{j=1}^{N} \frac{2 \rho_{j}}{\rho_{j}+\lambda}\right]=0 .
$$

Далее, нетрудно заметить, что в силу вытекающих из (41) неравенств $\rho_{n}>\rho_{n+1}, n=$ $1, \ldots, N-1$, числа $\lambda=-\rho_{j}, j=1, \ldots, N$, не являются решениями уравнения (44), а значит, оно эквивалентно уравнению

$$
\sum_{j=1}^{N} \frac{\rho_{j}}{\rho_{j}+\lambda}=-\frac{1}{2}
$$

Это уравнение имеет на полуоси $\lambda<0$ ровно $N$ различных корней, так как входящая в левую часть (45) функция на интервалах $\left(-\rho_{j},-\rho_{j+1}\right), j=1, \ldots, N-1$, и $\left(-\infty,-\rho_{1}\right)$ монотонно меняется в пределах от $-\infty$ до $+\infty$ и от 0 до $-\infty$ соответственно. 
Анализ уравнений (43) тривиален. Действительно, опираясь на формулы (39), (41), приходим к выводу, что

$$
\max _{m \geqslant N+1}\left(1-2 S-\nu_{0} \pi^{2} m^{2}\right)=\frac{1-\nu_{0} / \bar{\nu}_{N+1}}{2 N+1} .
$$

Отсюда очевидным образом следует, что состояние равновесия $O_{N}$ экспоненциально устойчиво (неустойчиво) при $\nu_{0}-\bar{\nu}_{N+1}>0\left(\nu_{0}-\bar{\nu}_{N+1}<0\right)$.

Покажем теперь, что каждое состояние равновесия $O \in K, O \neq O_{N}$, при условии (40) экспоненциально неустойчиво. Из структуры правых частей системы (36) вытекает, что любое такое состояние равновесия имеет лишь конечное число ненулевых координат $\rho_{n_{j}}=\eta_{j}>0, j=1, \ldots, k$, где $n_{1}<\cdots<n_{k}$ - некоторый фиксированный набор натуральных чисел, причем

$$
\eta_{j}=1-2 S-\nu_{0} \pi^{2} n_{j}^{2}, \quad j=1, \ldots, k, \quad S=\frac{1}{2 k+1}\left(k-\nu_{0} \pi^{2} \sum_{m=1}^{k} n_{m}^{2}\right)
$$

а требование $\min _{j} \eta_{j}>0$ эквивалентно выполнению неравенств

$$
0 \leqslant \nu_{0}<\nu_{*}=\left(\pi^{2}(2 k+1)\left(n_{k}^{2}-\frac{2}{2 k+1} \sum_{m=1}^{k} n_{m}^{2}\right)\right)^{-1} .
$$

В случае, когда $O=O_{n}$ при некотором $n \neq N$, факт неустойчивости состояния равновесия $O$ вытекает из проведенного выше анализа. Если же $O \neq O_{n}$ для любого $n$, то обязательно найдется такое $m \in \mathbb{N}$, что $m<n_{k}, m \neq n_{j}, j=1, \ldots, k$. Линеаризуем исходную систему (36) на этом состоянии равновесия и из получившейся системы в вариациях возьмем только одно уравнение для компоненты $h_{m}$ с указанным выше номером $m$. Нетрудно заметить, что данное уравнение имеет вид

$$
\dot{h}_{m}=\left(1-2 S-\nu_{0} \pi^{2} m^{2}\right) h_{m},
$$

а из формул (46), условия (47) и неравенства $m<n_{k}$ вытекает строгая положительность фигурирующей в (48) величины $1-2 S-\nu_{0} \pi^{2} m^{2}$. Теорема 3 доказана.

В дополнение к установленной теореме отметим, что при $\nu_{0}=\bar{\nu}_{N}$ состояние равновесия $O_{N}$ "влипает" в аналогичную неподвижную точку $O_{N-1}$, так как в этом случае обращается в нуль компонента $\rho_{N}$ из (41), а все остальные координаты $\rho_{n}$, $n=1, \ldots, N-1$, остаются положительными и совпадают с координатами точки $O_{N-1}$. Таким образом, при $\nu_{0} \rightarrow 0$ в системе (36) наблюдается цепочка бифуркаций вида $O_{1} \rightarrow \cdots \rightarrow O_{N} \rightarrow O_{N+1} \rightarrow \cdots$, которой в исходной задаче $(28)$ при согласованном стремлении параметров $\varepsilon$ и $\nu_{0}$ к нулю отвечает аналогичная цепочка бифуркаций устойчивых инвариантных торов. Точнее говоря, в силу теорем 2, 3 при фиксированном $\nu_{0}$, удовлетворяющем неравенствам (40), и при подходящем уменьшении $\varepsilon$ краевая задача (28) имеет устойчивый инвариантный тор любой наперед заданной конечной размерности. А это, собственно, и означает, что при $\varepsilon, \nu_{0} \rightarrow 0$ в ней реализуется сценарий развития турбулентности по Ландау. 
Одна из интересных особенностей краевой задачи (28) состоит в том, что при $0<\varepsilon \ll 1, \nu_{0}=0$ удается доказать наличие у нее так называемого турбулентного аттрактора. Причина, позволяющая это сделать, заключается в существовании при $\nu_{0}=0$ корректной замены переменных, приводящей исходную задачу (28) к системе вида

$$
\dot{\xi}=\Lambda_{0} \xi+\varepsilon \Lambda_{1}(\xi)+\varepsilon^{2} \Lambda_{*}(\xi, \varepsilon)
$$

в пространстве последовательностей $Z_{0}$. Здесь $\xi=\left(\xi_{1}, \bar{\xi}_{1}, \ldots, \xi_{n}, \bar{\xi}_{n}, \ldots\right) \in Z_{0}$,

$$
\begin{aligned}
\Lambda_{0} & =\operatorname{diag}\left\{i \omega_{1},-i \omega_{1}, \ldots, i \omega_{n},-i \omega_{n}, \ldots\right\}, \\
\Lambda_{1}(\xi) & =\left(\Lambda_{1,1}(\xi), \bar{\Lambda}_{1,1}(\xi), \ldots, \Lambda_{n, 1}(\xi), \bar{\Lambda}_{n, 1}(\xi), \ldots\right), \\
\Lambda_{n, 1}(\xi) & =\frac{1}{2}\left[1-3\left|\xi_{n}\right|^{2}-2 \sum_{\substack{k \geqslant 1, k \neq n}}\left|\xi_{k}\right|^{2}\right] \xi_{n}, \quad n \geqslant 1,
\end{aligned}
$$

а оператор $\Lambda_{*}(\xi, \varepsilon)$ обладает следующим свойством: для любого $m \in \mathbb{N}$ и произвольного ограниченного подмножества $B \subset Z_{0}$ найдется такое достаточно малое $\varepsilon_{0}=\varepsilon_{0}(m, B)>0$, что при каждом $\varepsilon \in\left[0, \varepsilon_{0}\right]$ этот оператор действует из $B$ в $Z_{0}$ и все его производные Фреше $\partial_{\xi}^{k} \Lambda_{*}(\xi, \varepsilon), k=0, \ldots, m$, непрерывны и ограничены по совокупности переменных $(\xi, \varepsilon) \in B \times\left[0, \varepsilon_{0}\right]$.

Действительно, предположим, что нужная замена переменных уже построена и задача (28) преобразована к виду (49), (50). Рассмотрим отвечающую (49) укороченную систему

$$
\dot{\xi}_{n}=i \omega_{n} \xi_{n}+\frac{\varepsilon}{2}\left[1-3\left|\xi_{n}\right|^{2}-2 \sum_{\substack{k \geqslant 1, k \neq n}}\left|\xi_{k}\right|^{2}\right] \xi_{n}, \quad n \geqslant 1,
$$

и выполним в ней последовательно замены $\xi_{n} e^{-i \omega_{n} t} \rightarrow \xi_{n}, n \geqslant 1, \tau=\varepsilon t$. В результате для $\rho_{n}=\left|\xi_{n}\right|^{2}$ получим в точности систему (36) при $\nu_{0}=0$, порождающую в инвариантном конусе $K \subset l$ полупоток $g^{\tau}(\rho), \tau \geqslant 0, \rho \in K$. Далее заметим, что в силу очевидного неравенства $d S(\tau) / d \tau \leqslant-1$ при $S(\tau) \geqslant 1$, где $S(\tau)=\sum_{n=1}^{\infty} \rho_{n}(\tau)$, множество $\mathcal{U}=K \cap\{\rho \in l:\|\rho\| \leqslant 1\}$ является для данного полупотока инвариантным и поглощающим. Тем самым в $K$ существует глобальный аттрактор

$$
\widetilde{\Omega}_{0}=\bigcap_{\tau \geqslant 0} g^{\tau}(\mathcal{U})
$$

Из проделанного при обосновании теоремы 3 анализа следует, что $\widetilde{\Omega}_{0}$ содержит счетное число неустойчивых состояний равновесия вида

$$
\begin{aligned}
\rho_{n_{j}} & =\frac{1}{2 k+1}, \quad j=1,2, \ldots, k ; \\
\rho_{n} & =0, \quad n \in \mathbb{N} \backslash\left\{n_{j}\right\},
\end{aligned}
$$

где $n_{1}<\cdots<n_{k}-$ произвольный конечный набор натуральных чисел. Кроме того, неустойчивое многообразие любого из состояний равновесия (53) является бесконечномерным и также принадлежит множеству (52). А это означает, что $\widetilde{\Omega}_{0}$ заведомо 
некомпактно и имеет бесконечную хаусдорфову размерность. Аттрактор с такими свойствами в дальнейшем будем называть турбулентным.

Следует отметить, что аналогичный (52) турбулентный аттрактор $\Omega_{0}$ существует и у системы $(51)$ : любой точке $\rho_{0}=\left(\rho_{1}^{0}, \ldots, \rho_{n}^{0}, \ldots\right)$ из $\widetilde{\Omega}_{0}$ отвечает совокупность векторов $\xi \in \Omega_{0}$ с компонентами $\xi_{n}=\sqrt{\rho_{n}^{0}} e^{i \varphi_{n}}, n \geqslant 1$, где вещественные параметры $\varphi_{n} \in[0,2 \pi]$ меняются независимо друг от друга. Добавим также, что состояниям равновесия (53) в укороченной системе (51) соответствуют неустойчивые инвариантные торы.

Возвращаясь к полной системе (49) и учитывая описанные выше свойства остатка $\Lambda_{*}(\xi, \varepsilon)$, нетрудно проверить, что для ее траекторий шар $B=\left\{\xi \in Z_{0}:\|\xi\| \leqslant 1\right\}$ оказывается инвариантным. Для этого достаточно взять функцию Ляпунова $V(\xi)=$ $\|\xi\|^{2}$ и убедиться, что

$$
\sum_{n=1}^{\infty} \frac{\partial V}{\partial \xi_{n}} \dot{\xi}_{n}+\frac{\partial V}{\partial \bar{\xi}_{n}} \dot{\bar{\xi}}_{n}=-\varepsilon-\varepsilon \sum_{n=1}^{\infty}\left|\xi_{n}\right|^{4}+O\left(\varepsilon^{2}\right)
$$

равномерно по $\xi:\|\xi\|=1$. Отсюда, очевидно, следует, что в шаре $B$ система (49) имеет асимптотически близкий к $\Omega_{0}$ турбулентный аттрактор $\Omega(\varepsilon)$. Более того, из свойств ограниченности и гладкости остатка $\Lambda_{*}(\xi, \varepsilon)$ вытекает существование для любого натурального $k$ такого $\varepsilon_{0}=\varepsilon_{0}(k)>0$, что при $\varepsilon \in\left(0, \varepsilon_{0}\right]$ аттрактор $\Omega(\varepsilon)$ содержит счетное число неустойчивых инвариантных торов, отвечающих состояниям равновесия (53) при фиксированном $k$ и при произвольном выборе номеров $n_{1}<\cdots<n_{k}$.

Итак, проблема существования турбулентного аттрактора свелась к отысканию упомянутой выше замены переменных. При построении этой замены удобнее работать не с самой краевой задачей (28), а с аналогичным (7) абстрактным уравнением в пространстве $E$ (см. (6)). При $\nu_{0}=0$ данное уравнение имеет вид

$$
\dot{w}=A_{0} w+\varepsilon A_{1} w+\varepsilon F(w, \varepsilon),
$$

где

$$
A_{0}=\mathcal{V}_{0} \mathcal{D}, \quad A_{1}=\operatorname{diag}\{0,1\}, \quad F=\left(0,-\varepsilon b\left(\mathcal{D}^{-1} w_{1}\right)^{5}-w_{2} \int_{0}^{1} w_{2}^{2} d x\right)^{\mathrm{T}} .
$$

Для уравнения (54) интересующая нас замена переменных задается равенством

$$
w=\Theta_{0} \xi+\varepsilon \Theta_{1}(\xi), \quad \xi \in Z_{0},
$$

где линейный оператор $\Theta_{0}$ и нелинейный оператор $\Theta_{1}$ действуют из $Z_{0}$ в $E$ по правилам

$$
\begin{aligned}
\Theta_{0} \xi & =\sum_{n=1}^{\infty}\left(\xi_{n}+\bar{\xi}_{n}, i \xi_{n}-i \bar{\xi}_{n}\right)^{\mathrm{T}} e_{n}(x), \\
\Theta_{1}(\xi) & =\sum_{n=1}^{\infty}\left(\theta_{n, 1}(\xi), \theta_{n, 2}(\xi)\right)^{\mathrm{T}} e_{n}(x),
\end{aligned}
$$

а коэффициенты $\theta_{n, 1}, \theta_{n, 2}$ определяются следующим образом. Обратимся к равенствам $(35)$ для $u_{n, 1}(t, \tau), n \geqslant 1$, и будем считать, что вместо $\xi_{n}(\tau), \xi_{k}(\tau), k \neq n$, в них 
подставлены координаты $\xi_{n}, \xi_{k}, k \neq n$, произвольного не зависящего от $\tau$ вектора $\xi \in Z_{0}$. В результате получаются формальные тригонометрические ряды, которые мы обозначим через $u_{n, 1}(t, \xi), n \geqslant 1$, подчеркивая их зависимость от $\xi$. Используя эти ряды, положим

$$
\theta_{n, 1}(\xi)=\omega_{n} u_{n, 1}(0, \xi), \quad \theta_{n, 2}(\xi)=\frac{\partial u_{n, 1}}{\partial t}(0, \xi), \quad n \geqslant 1 .
$$

Корректность приведенной замены вполне очевидна. В самом деле, в силу первого равенства в (56) линейный оператор $\Theta_{0}$ ограничен вместе со своим обратным, а из формул (35), (56), (57) вытекает, что нелинейность $\Theta_{1}(\xi)$ представляет собой ограниченную кубическую форму. Остается отметить, что, как показывает непосредственная проверка, замена (55) действительно преобразует уравнение (54) к требуемому виду (49).

\section{4. НЕЛОКАЛЬНЫЙ СЛУЧАЙ}

В данном разделе мы приведем результаты численного анализа аттракторов краевой задачи (2) при $\nu \rightarrow 0$ в случае, когда все остальные параметры имеют порядок единицы. Точнее говоря, всюду ниже мы считаем, что эти параметры фиксированы, в частности,

$$
\varepsilon=b=10, \quad a^{2}=0.1 .
$$

Для описания соответствующего численного эксперимента сначала, полагая $u_{t}=$ $v$, перейдем от уравнения второго порядка из (2) к системе

$$
\begin{gathered}
u_{t}=v, \quad v_{t}=\varepsilon v+\nu v_{x x}-v \int_{0}^{1} v^{2} d x+a^{2} u_{x x}-u-b u^{5}, \\
\left.u\right|_{x=0}=\left.u\right|_{x=1}=\left.v\right|_{x=0}=\left.v\right|_{x=1}=0 .
\end{gathered}
$$

Заменим в этой системе частные производные по $x$ и интегральное слагаемое приближенными равенствами

$$
\begin{aligned}
\left.u_{x x}(t, x)\right|_{x=k /(N+1)} & \approx(N+1)^{2}\left(u_{k+1}(t)-2 u_{k}(t)+u_{k-1}(t)\right), \\
\left.v_{x x}(t, x)\right|_{x=k /(N+1)} & \approx(N+1)^{2}\left(v_{k+1}(t)-2 v_{k}(t)+v_{k-1}(t)\right), \\
\int_{0}^{1} v^{2}(t, x) d x & \approx \frac{1}{N+1} \sum_{k=1}^{N} v_{k}^{2}(t),
\end{aligned}
$$

где $N$ - произвольно фиксированное натуральное число, $u_{k}(t)=\left.u(t, x)\right|_{x=k /(N+1)}$, $v_{k}(t)=\left.v(t, x)\right|_{x=k /(N+1)}, k=1, \ldots, N$. В результате для переменных $u_{k}, v_{k}$ приходим к конечномерной модели

$$
\begin{aligned}
& \dot{u}_{k}=v_{k}, \\
& \quad \dot{v}_{k}=\varepsilon v_{k}+\nu(N+1)^{2}\left(v_{k+1}-2 v_{k}+v_{k-1}\right)-\frac{v_{k}}{N+1} \sum_{m=1}^{N} v_{m}^{2}+ \\
& \quad+a^{2}(N+1)^{2}\left(u_{k+1}-2 u_{k}+u_{k-1}\right)-u_{k}-b u_{k}^{5}, \quad k=1, \ldots, N,
\end{aligned}
$$

где $u_{0}=v_{0}=u_{N+1}=v_{N+1}=0$. 
Компьютерный анализ модели (60) проводился при условиях (58) в диапазоне параметров $5 \leqslant N \leqslant 30,0 \leqslant \nu \leqslant 1.01$ с помощью пакета программ tracer 3.70, разработанного Д. С. Глызиным. Использовался метод Дорманда-Принса $8(5,3)$ с автоматическим выбором шага, величина которого во всех случаях не превосходила 0.001. Подчеркнем, что указанный метод, впервые приведенный в статье [13], в настоящее время является одним из стандартных методов численного решения дифференциальных уравнений и реализован, например, в таких пакетах, как MatLab, Dynamics Solver.

Обратимся к вопросу о поведении различных характеристик аттрактора $\Omega_{N}$ системы (60) при фиксированном $\nu \geqslant 0$ и при увеличении $N$. Оказывается, что в случае $\nu=0$ ляпуновская размерность $d_{\mathrm{L}}$ этого аттрактора при $N \rightarrow \infty$ неограниченно растет, а его старший ляпуновский показатель $\lambda_{\max }$, наоборот, убывает и при $N \geqslant 25$ становится равным нулю. Графики зависимости указанных величин от $N$ представлены на рис. 1 и 2 . Добавим также, что на промежутке $5 \leqslant N \leqslant 23$ для $d_{\mathrm{L}}$ справедлива приближенная формула $d_{\mathrm{L}} \approx 1.028+1.509 N$ (в этом случае аттрактор $\Omega_{N}$ оказывается хаотическим). В случае $N \geqslant 25$ множество $\Omega_{N}$ представляет собой $(2 N-3)$-мерный инвариантный тор, движения на котором близки к квазипериодическим.

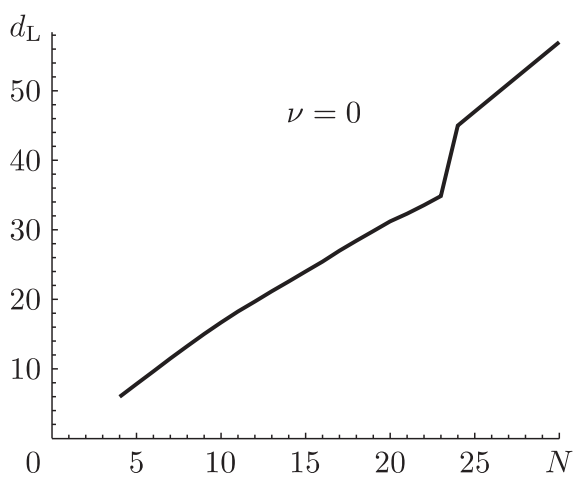

Рис. 1

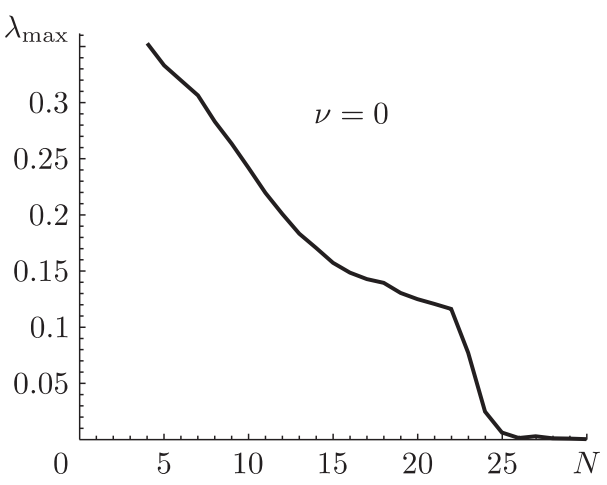

Рис. 2

При малых $\nu>0$ введенные выше величины $d_{\mathrm{L}}$ и $\lambda_{\max }$ с ростом $N$ ведут себя несколько иначе: при $N \rightarrow \infty$ они стремятся к конечным положительным пределам. Об этом свидетельствуют рис. 3-5, где изображены графики зависимости $\lambda_{\max }$ от $N$ для $\nu=0.001,0.002$ и 0.003 соответственно, а также рис. 6, на котором показана зависимость от $N$ размерности $d_{\mathrm{L}}$ в случае $\nu=0.002$.

Следует отметить, что при малых $\nu>0$ с ростом $N$ стабилизируется также сигнатура $\Sigma_{N}$ аттрактора $\Omega_{N}$, равная количеству его ненулевых положительных ляпуновских показателей. Например, при $\nu=0.002$ и при всех $N \geqslant 12$ выполняется равенство $\Sigma_{N}=3$.

Приведенные результаты численного расчета в совокупности с содержащимся в разделе 3 теоретическим анализом позволяют сделать следующие общие выводы о динамике по $\nu$ исходной распределенной модели (2). 


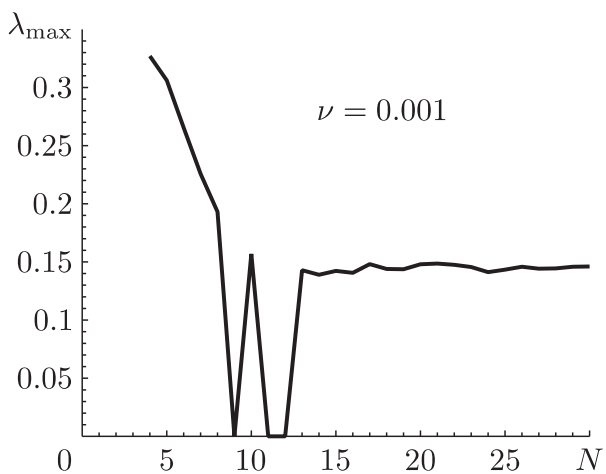

Рис. 3

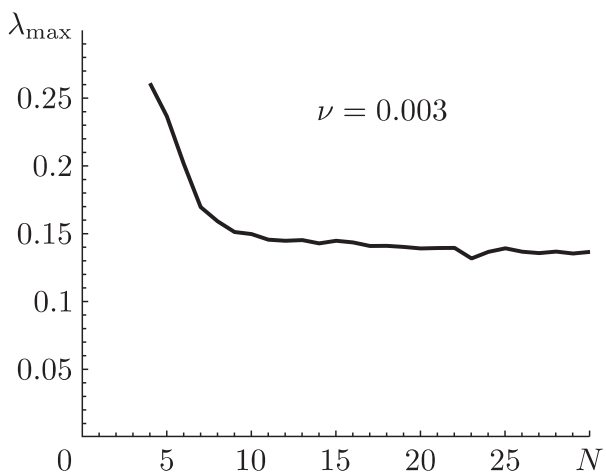

Рис. 5

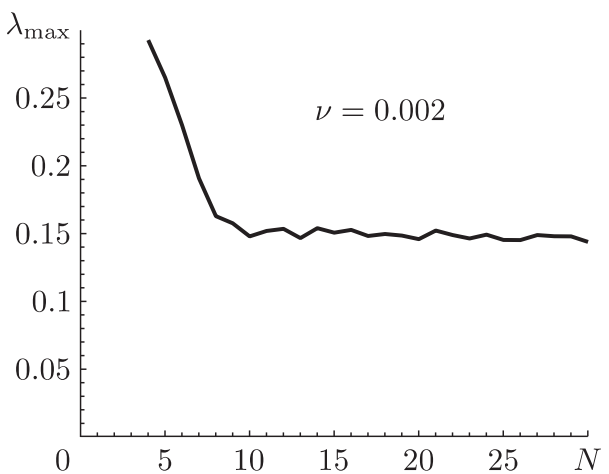

Рис. 4

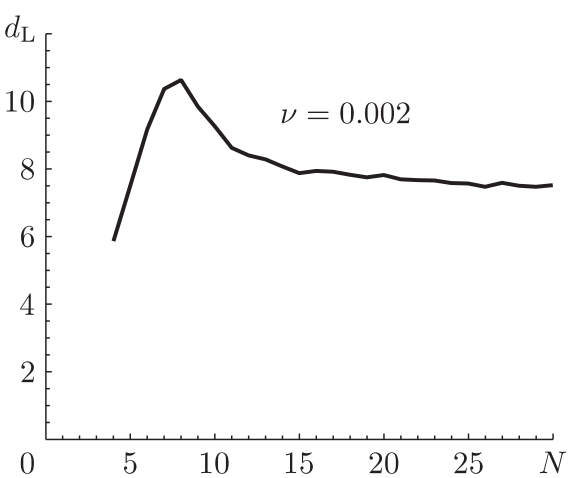

Рис. 6

1. При $\nu=0$ и при значениях параметров (58) у краевой задачи (2) существует турбулентный аттрактор $\Omega_{\text {turb }}$, который, по всей видимости, является продолжением по $\varepsilon$ аттрактора $\Omega(\varepsilon)$, обнаруженного в задаче $(28)$ при $0<\varepsilon \ll 1, \nu_{0}=0$. Как и $\Omega(\varepsilon)$, данный аттрактор представляет собой ограниченное и некомпактное множество в фазовом пространстве $\stackrel{\circ}{W} \frac{1}{2}(0,1) \times L_{2}(0,1)$, имеющее бесконечную хаусдорфову размерность. Кроме того, что оказалось весьма неожиданным, для “типичных" траекторий из $\Omega_{\text {turb }}$ справедливо равенство $\lambda_{\max }=0$. Таким образом, турбулентный аттрактор в предложенной нами модели можно отнести к так называемым странным нехаотическим аттракторам (см. [14]) или рассматривать как некий вариант "бесконечномерного тора".

2. Аттрактор $\Omega_{\text {turb }}$ является пределом при $\nu \rightarrow 0$ конечномерных хаотических аттракторов, ляпуновская размерность которых неограниченно растет. Последнее как раз означает, что при значениях параметров (58) и при уменьшении вязкости $\nu$ в краевой задаче (2) наблюдается интересующий нас сценарий развития турбулентности Ландау-Селла.

Дополнительным аргументом в пользу реализуемости упомянутого сценария служит визуальная информация, представленная на рис. 7, 8. На этих рисунках по- 


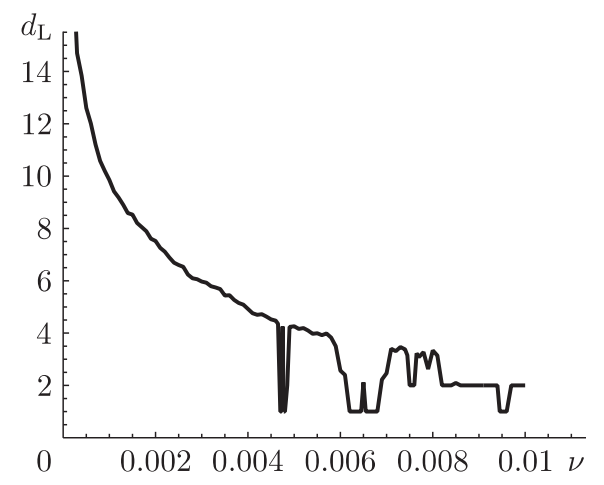

Рис. 7

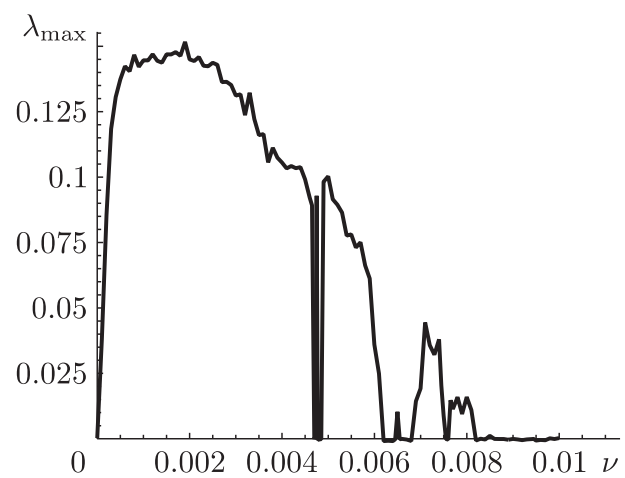

Рис. 8

казаны графики зависимости от $\nu$ величин $d_{\mathrm{L}}$ и $\lambda_{\max }$ аттрактора $\Omega_{N}$ системы $(60)$ при $N=30$, построенные на отрезке $0.005 \leqslant \nu \leqslant 0.01$ по точкам с шагом 0.0001 . Подчеркнем, что дальнейшее увеличение $N$ не имеет смысла, поскольку стабилизация характеристик $d_{\mathrm{L}}$ и $\lambda_{\max }$ происходит уже в случае $N=30$ (см. рис. $3-5$ ). Следовательно, есть все основания ожидать, что аналогичный вид имеют графики указанных характеристик и для аттракторов краевой задачи (2).

В заключение скажем несколько слов о бифуркациях, происходящих на начальных стадиях сценария Ландау-Селла. Анализ конечномерных моделей (60) позволяет сделать вывод, что в исходной распределенной модели (2) при значениях параметров (58) и при уменьшении $\nu$ наблюдаются следующие перестройки. При $\nu>10 / \pi^{2}$ устойчиво ее нулевое решение, а при прохождении $\nu$ через критическое значение $10 / \pi^{2}$ из нуля рождается устойчивый цикл $C$ (бифуркация Андронова-Хопфа). Далее этот цикл сохраняет устойчивость при $\nu_{*} \lesssim \nu<10 / \pi^{2}$, где $\nu_{*} \approx 0.03272$, а при последующем уменьшении $\nu$ отдает ее бифурцирующему из него двумерному тору $T$. Указанный тор, в свою очередь, существует и устойчив на промежутке $\nu_{* *} \lesssim \nu \lesssim \nu_{*}$, где $\nu_{* *} \approx 0.00812$, причем динамика на нем в некоторых случаях является периодической (например, она оказывается таковой при $\nu \in[0.00941,0.00967])$. Что касается хаотического аттрактора, то он впервые возникает в краевой задаче $(2)$ при $\nu \approx \nu_{* *}$ в результате разрушения тора $T$. Добавим, что наступлению стабильного хаоса, имеющего место при малых $\nu>0$, предшествует некоторое количество промежутков изменения $\nu$, в которых существуют устойчивые циклы. Наиболее крупные "окна периодичности” соответствуют значениям $\nu$ из интервалов [0.00476, 0.00483], [0.0062, 0.00648], [0.00655, 0.00686].

Благодарности. Работа выполнена при финансовой поддержке РФФИ (грант № 05-01-01004) и Целевой программы "Развитие научного потенциала высшей школы" (проект РНП.2.1.1.630).

\section{Список литературы}


[3] E. N. Lorenz, J. Atmos. Sci., 20:2 (1963), 130-141.

[4] D. Ruelle, F. Takens, Comm. Math. Phys., 20:3 (1971), 167-192.

[5] S. E. Newhouse, D. Ruelle, F. Takens, Comm. Math. Phys., 64:1 (1978), 35-40.

[6] П. Берже, И. Помо, К. Видаль, Порядок в хаосе. О детерминистском подходе к турбулентности, Меркурий-ПРЕСС, Череповец, 2000.

[7] M. J. Feigenbaum, J. Stat. Phys., 19:1 (1978), 25-52.

[8] Г. В. Осипов, Изв. вузов. Сер. радиофиз., 31:5 (1988), 624-627.

[9] G. R. Sell, "Resonance and bifurcations in Hopf-Landau dynamical systems", Nonlinear Dynamics and Turbulence, Interaction Mech. Math. Ser., eds. G.I. Barenblatt, G. Ioss, D.D. Joseph, Pitman, Boston, MA, 1983, 305-313.

[10] Д. Хенри, Геометрическая теория полулинейных параболических уравнений, Мир, М., 1985.

[11] А.Ю. Колесов, Н.Х. Розов, Инвариантные торы нелинейных волновых уравнений, Физматлит, М., 2004.

[12] Н.Н. Боголюбов, Ю.А. Митропольский, Асимптотические методы в теории нелинейных колебаний, Наука, М., 1974.

[13] J. R. Dormand, P. J. Prince, J. Comput. Appl. Math., 6:1 (1980), 19-26.

[14] В. С. Анищенко, В.В. Астахов, Т.Е. Вадивасова, А.Б. Нейман, Г. И. Стрелкова, Л. Шиманский-Гайер, Нелинейные эффекты в хаотических и стохастических системax, Ин-т компьютерных исследований, Москва-Ижевск, 2003.

Поступила в редакцию 14.03.2008 